\title{
PERCEPCIÓN DE ESTUDIANTES DE ODONTOLOGÍA SOBRE EL AMBIENTE EDUCATIVO DE LA FACULTAD Y EVALUACIÓN DEL DESEMPEÑO ACADÉMICO DEL DOCTORADO EN CIRUGÍA DENTAL
}

\section{Resumen}

Introducción. El propósito principal del estudio fue evaluar la percepción del estudiante de odontología en prácticas clínicas sobre el ambiente educativo que ofrece la Facultad de Odontología de la Universidad Evangélica de El Salvador UEES, así como evaluar los resultados del desempeño académico logrados durante su formación en la carrera del doctorado en cirugía dental.

Metodología. La investigación fue de tipo descriptivo, con diseño transversal y se realizó durante el período de enero a diciembre 2012. Se consideró evaluar el ambiente educativo por ser uno de los factores que determinan el alcance del éxito académico del estudiante a lo largo de su formación; a la vez, la medición de este factor, contribuye a establecer procesos de mejora contínua que le permiten al estudiante alcanzar su máximo desempeño académico, para su medición, se utilizó el cuestionario Dundee Ready Enviroment Evaluation Measurement DREEM por ser la herramienta más adecuada para evaluar ambientes de aprendizaje del área médica a nivel de pregrado. Este cuestionario y la revisión del historial académico de cada estudiante para la evaluación del desempeño académico se aplicaron a 71 estudiantes activos de odontología en prácticas clínicas. Resultados. La medición del ambiente educativo arrojó que los estudiantes de odontología perciben el ambiente de la Facultad como más positivo que negativo, en las áreas de: percepción del aprendizaje, percepción hacia los docentes, percepción del ambiente, así como la autopercepción social y académica.

\footnotetext{
1 Doctorado en Cirugía Dental, Máster en Docencia Universitaria, docente de salud pública y odontología preventiva de la Facultad de Odontología de la Universidad Evangélica de El Salvador, trabajo presentado para optar al título de Máster en Salud Pública.

2 Doctorado en Cirugía Dental, Vicedecana de la Facultad de Odontología de la Universidad Evangélica de El Salvador, trabajo presentado para optar al título de Máster en Salud Pública. e-mail: nadiamenjivar@yahoo.com

3 Doctor en Medicina, Epidemiólogo del Hospital Nacional Nuestra Señora de Fátima y docente de la Maestría en Salud Pública de la Universidad Evangélica de El Salvador.

4 Doctor en Medicina, Coordinador del Departamento de Salud Pública de la Facultad de Medicina y docente de la Maestría en Salud Pública de la Universidad Evangélica de El Salvador
} 
Percepción de estudiantes de odontología sobre el ambiente educativo de la facultad y evaluación del desempeño

Los resultados de la medición del desempeño académico indicaron que existen diferencias significativas entre los estudiantes cuando fueron evaluados por cada ciclo, así también, cuando se evaluó cada área académica; se demuestra un mayor desempeño académico en todas las asignaturas para el grupo de estudiantes que se encontraban inscritos en el ciclo XII, año 2012.

Conclusión. Los estudiantes perciben el ambiente educativo de la Facultad de Odontología como más positivo que negativo y existen diferencias significativas en el desempeño académico de los estudiantes, por grupos y por área académica. La correlación obtenida entre las notas por áreas académicas fué ligeramente positiva, mostrando que a medida el estudiante avanza también incrementa su rendimiento académico.

Palabras clave: desempeño académico, percepción del ambiente educativo, estudiante de odontología, El Salvador.

\section{Introducción}

La calidad en la educación superior se define como un concepto multidimensional de múltiples niveles, dinámico y que se relaciona con los elementos contextuales de un modelo educativo, con la misión y fin institucional, así como con estándares específicos dentro de un sistema, institución, programa o disciplina determinados. ${ }^{1,2}$

De la misma manera se reafirma la necesidad de preservar, reforzar y fomentar los valores fundamentales de la educación superior, tal como se sostuvo en la Conferencia Mundial sobre la Educación Superior, en la UNESCO en París, ya que estos valores contribuirán a la formación del recurso humano ${ }^{2}$. Así como existen sistemas de evaluación académica que permiten medir la calidad educativa, también deben garantizarse acciones concretas encaminadas al desarrollo de las potencialidades, los valores y las actitudes de los estudiantes. ${ }^{3,4,5}$

Para una institución formadora de profesionales en las áreas de la salud, el desarrollo de las competencias clínicas debe garantizar una combinación de habilidades, actitudes, aptitudes y conocimientos que provean la suficiente capacidad para emprender una tarea clínica específica. Para la rama de odontología, se especifican acuerdos internacionales tales como los documentados en la Asamblea General de la Federación Dental Internacional (FDI) de 2003 y los de la Asociación de Educación Dental Europea ADEE; con el mismo propósito, se crea la European Convergence in Dental Education (DentEdIII) que promueven la convergencia de planes de estudio armónicos y con mayores estándares de calidad. ${ }^{6,7}$

La formación del profesional integral demanda el desarrollo de las relaciones sustentadas mediante la integración de las asignaturas biomédicas, conductuales y las dentales con las cognitivas, psicomotoras y los valores éticos profesionales ${ }^{8}$ destacando el pensamiento crítico, el profesionalismo, la comunicación, la promoción de la salud, la evaluación del paciente, el diagnóstico y plan de tratamiento así como el establecimiento y mantenimiento de la salud oral como pilares fundamentales en la formación del recurso. ${ }^{7,12}$

Todos los elementos fundamentales integrados del proceso de enseñanza- 
Percepción de estudiantes de odontología sobre el ambiente educativo de la facultad y evaluación del desempeño

aprendizaje suceden dentro de un ambiente o clima educativo, por lo tanto este se considera como uno de los factores que determinan así mismo el éxito académico; su evaluación periódica es tan importante como la evaluación del desempeño académico y de la calidad educativa para la correcta valoración del éxito o fracaso de un programa de estudios. ${ }^{9}$

Existen algunos esfuerzos y un creciente interés, en el desarrollo de investigaciones orientadas al estudio de la percepción del estudiante en los ambientes educativos de las profesiones de la salud tales como medicina, odontología y enfermería ${ }^{10,11,12}$. Sin embargo, el estudio del ambiente y su influencia en la formación del profesional en odontología no ha contado con la suficiente atención como en el área médica, ${ }^{10}$ habiéndose desarrollado únicamente un instrumento específico para la evaluación de estudiantes en escuelas dentales en Australia en 2003. 9,11,12,13,16

La selección del instrumento a utilizar está basada en la idoneidad para medir el ambiente, en su validez y confiabilidad. Los educadores en salud han desarrollado un sin número de mediciones para examinar el entorno de aprendizaje y experiencias del estudiante; la herramienta de evaluación de ambiente educativo creada en la Universidad de Dundee, Escocia, Ilamada Dundee Ready Enviroment Evaluation Measurement ó DREEM por sus siglas en inglés, ha sido identificada como la herramienta más adecuada para evaluar ambientes de aprendizaje del área médica en niveles de pregrado. Este cuestionario ha sido catalogado como el más válido y fidedigno para evaluar ambientes completos o parciales. $9,11,12,13,14,15$
Considerando la importancia de las evaluaciones durante la formación del recurso humano en salud para el desarrollo de las máximas capacidades del estudiante, se definió como propósito del estudio, evaluar tanto el desempeño académico del estudiante, como su percepción sobre el ambiente educativo dentro de la Facultad de Odontología UEES durante en el período de enero a diciembre de 2012.

El programa de estudios del doctorado en cirugía dental vigente, comprende tres áreas de ciencias: básica, preclínica y clínica, siendo prerrequisito una de la otra para alcanzar el siguiente nivel de conocimientos con una nota mínima de aprobación de 6.0, en una escala de 0 a 10.

El estudiante de odontología permanece dentro del campus universitario durante catorce ciclos académicos (I-XIV); las prácticas clínicas se desarrollan durante los últimos cinco ciclos (X-XIV); el programa completo está diseñado para siete años, según lo establece el Ministerio de Educación de El Salvador para el doctorado en cirugía dental.

\section{Metodología}

La presente investigación es de tipo descriptivo con diseño transversal. La población fue de 71 estudiantes activos del doctorado en cirugía dental quienes durante el año 2012 cursaron al menos un semestre de asignaturas del área clínica, los cuales pertenecían a los ciclos X, XII y XIV. Las variables estudiadas fueron: desempeño académico, área académica, asignaturas trazadoras y la percepción del ambiente educativo. Los datos sobre el desempeño académico de cada estudiante se obtuvieron a través la nota final obtenida por el estudiante desde el inicio de la 
Percepción de estudiantes de odontología sobre el ambiente educativo de la facultad y evaluación del desempeño

carrera hasta el momento del estudio, estos datos fueron trasladados a una tabla de recolección en Excel. Las áreas académicas están determinadas por el plan de estudios vigente, y son: básica, preclínica y clínica, en cada una de éstas se eligieron aquellas asignaturas determinantes cuyos contenidos en conjunto o de forma individual son la base fundamental para cursar asignaturas del área académica superior.

La encuesta DREEM que se utilizó en la evaluación de la percepción del ambiente educativo es un cuestionario de 50 preguntas cerradas. Estas preguntas caen dentro de 5 subescalas: percepción del estudiante hacia el aprendizaje (12 enunciados); percepción de los estudiantes hacia los docentes (11 encunciados); autopercepción académica de los estudiantes (8 enunciados); percepción del estudiante sobre el ambiente (12 enunciados) y autopercepción social del estudiante (7 enunciados).

Cada una de las preguntas o enunciados se califica con un puntaje del 0 al 5 en las siguientes opciones: "Totalmente de acuerdo"(4), "De acuerdo"(3), "No estoy seguro"(2), algunos autores usan también para este puntaje medio, como "No sabe"; "En desacuerdo" (1) y "Totalmente en desacuerdo" (0). Los puntajes de las preguntas $4,8,9,17,25,35,39,48$ y 50 se valoran a la inversa por ser enunciados en negativo. Además, los puntajes resultantes más altos indicarán una evaluación más positiva. El cuestionario tiene un puntaje máximo por encuesta de 200 puntos, el cual representa un ambiente educativo ideal. ${ }^{10,12,13,29}$

Los resultados de la DREEM pueden ser evaluados de la siguiente manera: individual, por sub-escalas ó como DREEM general. Los puntajes netos obtenidos en cada enunciado son agrupados dentro de la escala a la que pertenecen y son sumados por cada estudiante encuestado, luego las medias de estos resultados son tomadas para establecer totales por sub-escala. ${ }^{29}$

Para la evaluación DREEM general se sumaron todos los puntajes individuales y se multiplicaron por el número de participantes (71), con este total se estableció una escala de cuatro rangos de valores que correspondieran a las categorías establecidas para el ambiente educativo $(A E)$, el máximo puntaje posible a obtener en este estudio era de 14,200, dividiéndose de la siguiente manera: AE muy pobre (03550), AE muy problemático (3551-7100), $A E$ más positivo que negativo (7101$10,650)$ y AE excelente $(10,651-14,200)$. Para las sub-escalas se aplicó la misma dinámica, tomando los puntajes de los enunciados correspondientes a cada categoría evaluada, de esa manera la percepción hacia el aprendizaje fue de la siguiente manera: $A E$ muy pobre, para el rango de (0-850), AE muy probllemático (851-1704), AE más positivo que negativo (1705-2556) o $\mathrm{AE}$ excelente (2557-3408). ${ }^{10,12,13}$

Para el análisis de datos se empleó el programa Microsoft Excel 2010, y el paquete estadístico computacional para ciencias sociales (SPSS) versión 15.0., utilizando estadística descriptiva para la variable de ambiente educativo, y para el desempeño académico se aplicó estadística inferencial con los test estadístico ANOVA, prueba de homogeneidad de varianza (Levene), post hoc: Tamhane, para comparar la media, y para establecer la correlación del rendimiento académico según área se utilizó la prueba de coeficiente de correlación de Pearson. 
Percepción de estudiantes de odontología sobre el ambiente educativo de la facultad y evaluación del desempeño

\section{Resultados}

La evaluación del ambiente educativo a través del cuestionario DREEM realizado a 71 estudiantes brindó un total general de 9391 puntos, definiendo el ambiente educativo (AE) como más positivo que negativo. Los puntajes obtenidos en cada sub-escala fueron: percepción del aprendizaje 2157, percepción hacia los docentes 2098, autopercepción social 1237, autopercepción académica 1669, percepción del ambiente 2230. (Tabla 1)

Tabla 1. Preguntas y valores obtenidos del cuestionario DREEM sobre la percepción hacia el aprendizaje de los estudiantes del doctorado de cirugía dental en área clínica de la Universidad Evangélica de El salvador, Enero Diciembre 2012.

\begin{tabular}{|c|c|c|c|}
\hline \# & $\begin{array}{l}\text { Pregunta de percepción hacia el apren- } \\
\text { dizaje }\end{array}$ & Puntaje & $\%$ \\
\hline 22 & $\begin{array}{l}\text { La enseñanza contribuye al desarrollo } \\
\text { de mi confianza }\end{array}$ & 216 & 81,8 \\
\hline 16 & $\begin{array}{l}\text { La enseñanza contribuye al desarrollo } \\
\text { de mis competencias }\end{array}$ & 210 & 79,5 \\
\hline 20 & La enseñanza está bien enfocada & 205 & 77,7 \\
\hline 47 & $\begin{array}{l}\text { Se prioriza el aprendizaje a largo plazo } \\
\text { en lugar del aprendizaje a corto plazo }\end{array}$ & 201 & 76,1 \\
\hline 25 & $\begin{array}{l}\text { La enseñanza pone demasiado énfasis } \\
\text { en el aprendizaje de detalles }\end{array}$ & 200 & 75,8 \\
\hline 38 & $\begin{array}{l}\text { Tengo claros los objetivos de aprendi- } \\
\text { zaje de mis cursos }\end{array}$ & 200 & 75,8 \\
\hline 1 & $\begin{array}{l}\text { Me siento motivado a participar en } \\
\text { clase }\end{array}$ & 198 & 75,0 \\
\hline 24 & $\begin{array}{l}\text { El tiempo que se destina a la enseñan- } \\
\text { za es bien utilizado }\end{array}$ & 192 & 72,7 \\
\hline 44 & $\begin{array}{l}\text { La enseñanza me anima a ser un estu- } \\
\text { diantes activo }\end{array}$ & 189 & 71,6 \\
\hline 13 & $\begin{array}{l}\text { La enseñanza está centrada en el es- } \\
\text { tudiante }\end{array}$ & 188 & 71,2 \\
\hline \multirow[t]{2}{*}{7} & $\begin{array}{l}\text { La enseñanza es frecuentemente esti- } \\
\text { mulante }\end{array}$ & 158 & 59,8 \\
\hline & Total puntaje obtenido & 2157 & \\
\hline
\end{tabular}

Fuente: datos obtenidos del estudio

Definiendo el ambiente educativo (AE) en cada sub escala en la categoría de más positivo que negativo. Los resultados muestran cómo la autopercepción social y la autopercepción académica son los menores puntajes obtenidos.(Figura 1)

Figura No 1. Puntajes totales obtenidos en cada sub-escala sobre la percepción del ambiente educativo (AE) de los estudiantes del doctorado de cirugía dental en área clínica de la Universidad Evangélica de El Salvador, Enero a Diciembre 2012.

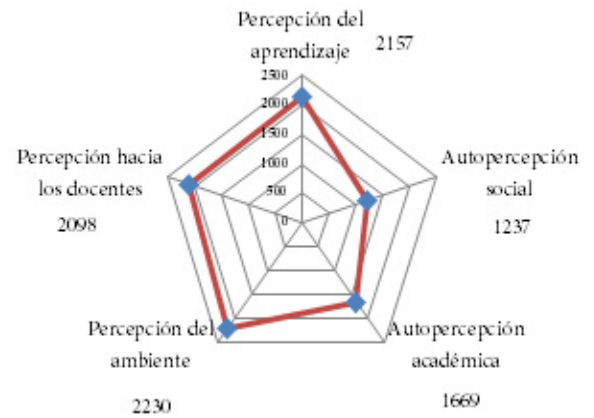

Fuente: datos obtenidos del estudio.

La evaluación del desempeño académico para cada grupo de estudiantes mostró que las medias en las notas obtenidas fue de: 7.12 para X ciclo, 7.58 para XII ciclo y 7.19 para XIV ciclo. Al evaluar el desempeño en cada área académica se encontraron las siguientes medias: 7.31 para área básica, 7.29 para preclínica y 7.89 para clínica. (Figura No 2).

Figura No 2. Medias del desempeño académico por área de los estudiantes del doctorado de cirugía dental en área clínica de la Universidad Evangélica de El Salvador, Enero a Diciembre 2012.

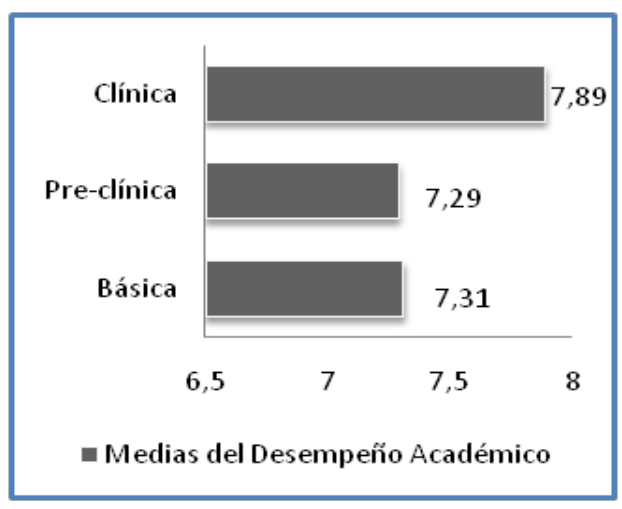

Fuente: datos obtenidos del estudio 
Percepción de estudiantes de odontología sobre el ambiente educativo de la facultad y evaluación del desempeño

La evaluación de las asignaturas trazadoras arrojó que los puntajes más bajos se encontraron en: fisiología humana 6.7, farmacología y terapéutica 6.97, operatoria dental II 6.74 y clínica de periodoncia I con 7.28; los mayores puntajes se reflejaron en las asignaturas de: biología y bioquímica con 7.58, anatomía de cabeza y cuello y neuroanatomía con 7.73, clínica de operatoria I 8.20, clínica de cirugía bucal 8.17 y la clínica de prostodoncia I con 8.05. Al analizar las medias con la prueba post-hoc Tamhane se mostró diferencia estadística significativa al evaluar la asignatura clínica de diagnóstico bucal I la cual presentó una media mayor que las asignaturas predecesoras como: microbiología médica, anatomía humana, fisiología humana, farmacología y terapéutica, semiología, de igual forma la clínica de cirugía bucal I presenta el mismo patrón con las asignaturas mencionadas. Al aplicar la prueba de coeficiente de correlación de Pearson, se mostró una ligera correlación positiva entre las variables área académica y la ponderación numérica.

\section{Discusión}

Los datos obtenidos en la evaluación del desempeño académico permitieron observar el rendimiento de los estudiantes desde el inicio de su carrera a través de las áreas básica, preclínica y clínica, a su vez, se compararon los resultados entre áreas académicas, entre grupos de estudiantes de diferentes niveles de clínicas, así como de manera individual; en todos los casos se demostró que las notas finales incrementan en valor a medida el estudiante avanza a cada área de mayor dificultad. Los resultados de las medias para las asignaturas trazadoras de las áreas básica y pre-clínica como fisiología humana, farmacología y terapéutica y operatoria dental II, arrojaron medias inferiores a 7, en contraste con la evaluación del área clínica donde la media supera el 8, reflejando una ligera correlación positiva. Este patrón, en el cual el rendimiento académico de las asignaturas altamente teóricas del área básica y preclínica presentan menores puntuaciones con respecto a las prácticas clínicas encontrado en este estudio, también se observa en otras universidades en estudios similares ${ }^{17,18}$

Por otro lado, el estudio del desempeño por grupos muestra que los mayores puntajes fueron obtenidospor losestudiantes inscritos en el ciclo XII al momento del estudio, su alto rendimiento se observa constante desde el área básica, manteniéndose con la misma tendencia a lo largo de su avance en las áreas de la pre-clínica y clínica.

Las discrepancias demostradas entre grupos y asignaturas, evidencia la complejidad en el cumplimiento de los propósitos de un plan de estudio, donde queda clara la influencia de diversos factores durante el proceso de desarrollo que inician desde el diseño curricular, los modelos didácticos, actúan las competencias del personal docente, para luego combinarse con los diversos estilos de aprendizaje de los estudiantes, que en conjunto inciden directa e indirectamente en todo el proceso de enseñanza aprendizaje de cualquier profesión en la que se desarrolle. ${ }^{19}$

Se debe destacar el compromiso que las escuelas formadoras tienen en relación a la implementación de estrategias innovadoras que promuevan el razonamiento crítico, fomentando la comprensión y la interacción con el entorno, así como la formulación y resolución de problemas clínicos, con el único propósito que el rendimiento académico que se refleja en este tipo de estudios sea fidedigno y sea resultado de 
Percepción de estudiantes de odontología sobre el ambiente educativo de la facultad y evaluación del desempeño

un proceso que facilita al estudiante el desarrollo de sus habilidades para la toma decisiones diagnósticas y terapéuticas en beneficio de sus pacientes.

Estos objetivos se verán potenciados al dar la importancia debida a la influencia del ambiente educativo en el éxito del desarrollo del profesional, con acciones que promuevan a su vez que tanto la institución como el facilitador desarrollen ese ambiente propicio haciendo el uso adecuado de los canales de comunicación, estimulando el uso de las tecnologías de la información, fomentando la adecuada expresión en público, impulsando una excelente gestión de conflictos grupales, entre otras habilidades que puedan favorecer de forma significativa el desempeño de todos los profesionales. ${ }^{19,20,21}$

A su vez, la estandarización de los sistemas de evaluación es fundamental para garantizar los resultados del proceso de enseñanzaaprendizaje donde se articulen las funciones de docencia, investigación y extensión o proyección social, de manera que el estudiante integre durante su formación las competencias cognoscitivas, habilidades y actitudes en general. ${ }^{17,22}$

La evaluación de la percepción del ambiente educativo brinda la oportunidad al estudiante de verter sus opiniones sobre su perspectiva hacia los docentes, sobre la enseñanza, del ambiente, del desarrollo social y de su propio aprendizaje; los resultados reflejan que la facultad ha logrado fomentar en ellos la empatía, reconocen que las bases adquiridas en cada nivel de estudios son fundamentales para su desempeño en niveles más complejos de la carrera, sin embargo, muestran no aplicar nuevas técnicas de estudio y tener dificultad en memorizar o retener todo el contenido de las asignaturas que reciben.

Varios autores han reportado que los hábitos, técnicas y actitudes hacia el estudio, son aspectosqueinfluyen significativamente enel rendimiento académico de los estudiantes, y particularmente, la motivación por aprender influye, no sólo en el éxito académico sino también en su desempeño profesional. Si bien el estudio es un factor importante para el éxito académico, también la organización personal del estudiante (cómo estudiar, dónde estudiar, cuándo estudiar) posibilita una buena calidad de aprendizaje, el cual sólo se logra mediante la comprensión de los conocimientos, para ello es necesario que el estudiante adquiera habilidades para utilizar métodos y técnicas de estudio eficaces. ${ }^{23}$

El estudio de la percepción hacia los docentes reflejó que el estudiante reconoce el dominio y la experiencia del docente al impartir las asignaturas y que las clases están orientadas al conocimiento del docente. El ambiente educativo es agradable y permite el desarrollo de habilidades interpersonales con sus compañeros. Sin embargo, se muestra conciencia de acciones para combatir el estrés, espacios para descanso y la oportunidad de interaccionar con otras facultades. ${ }^{9}$

Algunos autores consideran que el trato al estudiante es un factor clave para que su desempeño sea adecuado, así también que el docente sea el facilitador del conocimiento colocando al estudiante como centro del objetivo de la enseñanza. Lizzio et al en $2002^{9}$, encontraron que las percepciones de los estudiantes sobre su entorno educativo, emocional y social son fuertes factores predictivos en el desempeño estudiantil a nivel universitario más que los logros a 
Percepción de estudiantes de odontología sobre el ambiente educativo de la facultad y evaluación del desempeño

nivel de bachillerato ${ }^{13,24}$ Los ambientes de aprendizaje favorables generan en el estudiante la participación activa, permitiendo al estudiante comprender y transformar el conocimiento en un aprendizaje significativo, proporcionándole herramientas para resolver problemas de una forma crítica y creativa. ${ }^{25,26,27,28}$

\section{Conclusiones}

Los estudiantes perciben el ambiente educativo de la Facultad de Odontología como más positivo que negativo según los resultados obtenidos al evaluar las percepciones del aprendizaje, hacia los docentes, del ambiente, así como la autopercepción social y académica.

Los resultados más altos de la evaluación de percepción se reflejan en las áreas del aprendizaje y la auto percepción académica; estos reflejan el reconocimiento del estudiante en que la UEES fomenta su sensibilidad social, desarrollo de su confianza y aprendizaje de competencias pertinentes para su formación profesional y la calidad del cuerpo docente.

Los puntajes más bajos se encuentran en el área de la autopercepción social donde se señala la necesidad fomentar más acciones para apoyar al estudiante en el manejo del estrés, administración del tiempo, y falta de motivación; de la misma manera la relación del docente hacia el estudiante indica principalmente un enfoque de la enseñanza centrado hacia el docente, con la necesidad de abrir más espacios de participación y altos niveles de estrés.

Existen diferencias significativas en el desempeño académico de los estudiantes evaluados por grupos y por área académica, mostrando el desempeño más alto en todas las asignaturas del pensum académico los estudiantes del nivel XII.

La correlación obtenida entre las notas de todas las áreas fue ligeramente positiva, mostrando que a medida el estudiante avanza a lo largo de su carrera también su rendimiento académico. El grupo de asignaturas contempladas dentro del área clínica mostró ponderaciones superiores en cuanto a las medias con respecto a las asignaturas de los niveles básico y preclínico.

\section{Recomendaciones}

Desarrollar una herramienta de evaluación para las asignaturas clínicas que le permita al docente ponderar no sólo el dominio de conocimientos teóricos o prácticos sino también incorporar la capacidad de análisis y resolución de problemas por parte del estudiante.

Proponer un plan de estudios con énfasis en el aprendizaje por competencias, fomentando así en el futuro profesional el desarrollo del análisis crítico necesario para afrontar los retos profesionales de la actualidad.

Fomentar el desarrollo profesional integral a través de la generación de espacios de participación social, artística, deportiva y espiritual intra e interfacultades que le permitan interactuar socialmente, así como programas de apoyo que le ayuden al estudiante a manejar adecuadamente el alto nivel de estrés generado por su carrera.

Integrar temas como técnicas de estudio, gestión del tiempo, priorización de tareas, a cátedras clave, que sirvan de apoyo para desarrollar sus máximos potenciales de rendimiento académico. 
Percepción de estudiantes de odontología sobre el ambiente educativo de la facultad y evaluación del desempeño

Mantener el nivel de especialización y estimular la experiencia de los docentes, orientando sus conocimiento a la aplicación de las tecnologías de información y al aprendizaje basado en competencias.

Establecer un plan de mejora, sobre el rendimiento académico y la percepción del ambiente educativo a fin de garantizar el progreso de las áreas que presentaron menores puntuaciones en este estudio.

Realizar un estudio de la misma naturaleza en cada una de las facultades de la UEES, de tal manera que sirva de apoyo a mejorar los niveles de satisfacción del estudiante y a los planes de mejora contínua de la calidad a nivel institucional.

\section{Fuentes de información consultadas}

1. Alcantar Armando Santuario Dimensiones de la calidad superior reencuentro, diciembre, numero 050 Universidad Autónoma Metropolitana-Xochimilco, Distrito Federal México pp 20-21, [en línea] 2007[Fecha de acceso 20 noviembre de 2012]. URLDisponible:http://redalyc.uaemex.mx/pdf/340/34005004.pdf.

2. Organización de Las Naciones Unidades para la Educación (UNESCO) declaración mundial sobre la educación superior en el siglo XXI: visión y acción 9 de octubre de 1998 [en línea]. [Fecha de acceso 15 de octubre de 2012.] Disponible en: http://www.unesco.org/ education/educprog/wche/declarationspa. htm

3. Organización Panamericana de la Salud (OPS). La Renovación de la Atención Primaria de Salud en las Américas No. 3 La Acreditación de Programas de Formación en Medicina y la orientación hacia la APS, Washington D.C., Mayo, 2010 [en línea]. . [Fecha de acceso 17 noviembre de 2012] URL disponible en http:// www2.paho.org/hq/dmdocuments/2010/HSSSeries-APS-3-Acreditacion.pdf.
4. Cabrero García, Benilde. Modelos teóricos e Indicadores de Evaluación Educativa. sinéctica [en línea]. 2010, [en línea]. n.35, pp 1-17. ISSN 1665-109X. [Fecha de acceso 14 Noviembre de 2012] URL disponible en http:// www.scielo.org.mx/scielo.php?script=sci_artte xt\&pid=S1665109X2010000200005.

5. Calatrava Oramas Luis Alonso Educación por Competencias En Odontología Acta Odontológica Venezolana - 2010 [en línea] Volumen 48 № 1 / ISSN: 0001-6365 - [Fecha de acceso 30 noviembre de 2012]

6. Da Silva, Erica Tatiane Fátima Nunes Maria de, Goretti Queiroz Maria, R. Leles Cláudio Factors Influencing Students' Performancein a Brazilian Dental School Braz Dent J (2010) 21(1): 80-86 ISSN 0103-6440 Dental School, Federal University of Goiás, Goiânia, GO, Brazil.

7. Commission on Change and Innovation in Dental Education (ADEA). Competencies for the general dentist September 12, $2006 \mathrm{El}$ entorno dental Educación ADEA Comisión de Cambio y la Innovación en la Educación Dental Educ Dent J. 70: 1265-1270

8. Genn J. AMEE Medical Education Guide No. 23 (Part 1): curriculum,environment, climate, quality and change in medical education- a unifying perspective. Med Teach 2001: 23: 337-344

9. L.A. Foster Page, M. Kang, V. Anderson and W.M. Thomson. Appraisal of the Dundee Ready Educational enviroment measure in the New Zealand dental educational environment. European Journal of Dental Education.Volume 16, Issue 2 (2012) pag 78-85. Published online 08 Dic. 2011.

10. S. Ostapczuk, A. Hugger, J. de Bruin, S. RitzTimme, and T. Rotthoff. DREEM on, dentists Students' perceptions of the educational environment in a German dental school as measured by the Dundee Ready Education Environment MeasureEuropean Journal of 
Percepción de estudiantes de odontología sobre el ambiente educativo de la facultad y evaluación del desempeño

Dental Education ISSN 1396-5883 Eur J Dent Educ 16 (2012) 67-77.

11. Divaris K, Barlow PJ, Chendea SA, et al. The academic environment: the students' perspective. Eur J Dent Educ 2008: 12(Suppl.1): $120-130$

12. Soemantri D., Herrera C., Riquelme A. Measuring the educational environment in health professions studies: a systematic review. Med Tech 2010: 32: 947-952.

13. McAleer S, Roff S. A practical guide to using the Dundee Ready Education Environment Measure (DREEM). In: Genn JM ed. Curriculum,environment, climate, quality and change in medical education:A unifying perspective. AMEE Education Guide No. 23.Dundee: Association for Medical Education in Europe, 2001: 23.(28)

14. Gerzina TM, McLean T, Fairley J. Dental clinical teaching: perceptionsof students and teachers. J Dent Educ 2005: 69: 1377-1384.

15. Miles S, Leinster SJ. Medical students' perceptions of their educational environment: expected versus actual perceptions.Med Educ 2007: 41: 265-272.

16. Herrera Cristian et al.Evaluación del ambiente educacional pre-clínico en seis escuelas de medicina en Chile Rev Med Chile ;2010 138: 677-684

17. Moromi Nakata, Hilda La Influencia de la ejecución curricular y el uso de medios y materiales en el rendimiento académico de los estudiantes de la Facultad de Odontología de la Universidad Nacional Mayor de San Marcos. . [en línea] [fecha de acceso 1 diciembre 2012]Disponible:http://biblioteca. universia.net/html_bura/ficha/params/title/ influenciaejecucioncurricular-uso-mediosmateriales-rendimientoacademicoestudiantesf acultad/id/34694034.html
18. Pinto Natalia, Kader Nevertiti, Pineda Xenia, Acuña Edward. Rendimiento académico estudiantil y su relación con el régimen de estudio: Facultad de Odontología LUZ. Ciencia Odontológica [en línea ]. 2007 [citado 2012 Dic 11] ; 4(1): 7-22. URL Disponible en: http:// www2.scielo.org.ve/scielo.php?script=sci_artt ext\&pid=S131782452007000100002\&lng=es.

19. Nora Haghparast D.D.S., Masakazu Okubo D.D.S.,PhD Reyes Enciso M.S.Ph.D, Glenn T. Clark D.D.S., M.S. y Charles Shuler D.M.D. Ph.D. Comparing student-generated learning needs with faculty objectives in PBL cases in dental education DentalDent J Educ 2011 v 75 No 8, pags $1092-1097$. August, 2011.

20. Shiva Khatami y Michael I. MacEntee Evolución del razonamiento clínico en Educación Dental Dent J Educ 2011 vol 75 : $321-328$

21. Marcos Scarbecz, Cynthia K. Russell, Robert G. Shreve ,Melissa M. Robinson, y Cheryl R. Scheid. Capacitación Docente para mejorar la enseñanza en un Centro de Ciencias de la Salud: Una Evaluación de Necesidades. J Dent Educ 201175 : 145 -159.

22. Aravena Verdejo Víctor, García-Hernández, Fernando Anatomía y Competencias Clínicas en Odontología.Estudio Basado en Apreciación de Académicos Anatomy and Clinical Competences in Dentistry. Study Based on Academics Appreciation Int. J. Morphol., 24(4):713-720, 2006.

23. Mena, Analía; Golbach Marta; Véliz, Influencia de los hábitos de estudio en el rendimiento de alumnos ingresantes Margarita Facultad de Ciencias Económicas. Universidad Nacional de Tucumán Provincia de Tucumán (Argentina) 2012 [en línea ]. fecha de acceso 1 diciembre] URL Disponible en:http://www.soarem.org.ar/ Documentos/48\%20Mena.pdf.

24. Henzi D, Davis E, Jasinevicius R, Hendricson W. North American dental students' perspectives about their clinical education. I DentEduc 2006: 70: 361-377 
Percepción de estudiantes de odontología sobre el ambiente educativo de la facultad y evaluación del desempeño

Ethel Mireya López Pérez, Nadia María Menjívar Morán, Mauricio Antonio Abarca, Humberto Alcides Urbina,

págs. 28-38.

25. Zamzuri AT, Azli NA, Roff S, McAleer S. How do students at dental training college Malaysia perceived their educational environment? Malaysian Dent J 2004: 25: 15-35

26. Herrera Soemantri D, C, Riquelme A. Measuring the educational environment in health professions studies: a systematic review. MedTeach 2010: 32: 947-952.(37)

27. Celso Zilbovicius, Maria Ercilia de Araujo,Carlos Botazzo, Antonio Carlos Frías, Simone Rennó Junqueira, y Cilene Rennó Junqueira Un cambio de paradigma en los currículos Dental Predoctoral en Brasil: Evaluación del proceso de cambio Dent J Educ 201175 : 557 - 564

28. Treviño de la Garza Mirta Concepción, Noriega Luna Leticia Imelda Ambientes favorables para el aprendizaje y para el desarrollo de competencias.[fecha de acceso 1 diciembre] URL Disponible en: http://e-cademic.sems. udg.mx/formacion_docente_e_investigacion/ investigacion/publicaciones/docucoloq37.pdf 